ON THE GENERIC FINITENESS OF EQUILIBRIUM OUTCOME DISTRIBUTIONS IN GAME FORMS

by

Srihari Govindan and Andrew McLennan

Discussion Paper No. 299, April 1997

Center for Economic Research

Department of Economics

University of Minnesota

Minneapolis, MN 55455 


\title{
On the Generic Finiteness of Equilibrium Outcome Distributions in Game Forms*
}

\author{
by \\ Srihari Govindan \\ Department of Economics \\ University of Western Ontario \\ London, Canada N6A 5C2 \\ govindan@sscl. uwo.ca \\ and \\ Andrew McLennan \\ Department of Economics \\ University of Minnesota \\ 271 19th Avenue South \\ Minneapolis, MN 55455 \\ mclennan@atlas. socsci.umn.edu
}

April, 1997

* We would like to acknowledge stimulating conversations with Larry Blume, Andreu Mas-Colell, and Bill Zame. Govindan would like to thank the Social Sciences and Humanities Research Council of Canada for research support. 


\begin{abstract}
Consider nonempty finite pure strategy sets $S_{1}, \ldots, S_{n}$, let $S=S_{1} \times \ldots \times S_{n}$, let $\Omega$ be a finite space of "outcomes," let $\Delta(\Omega)$ be the set of probability distributions on $\Omega$, and let $\theta: S \rightarrow \Delta(\Omega)$ be a function. We study the conjecture that for any utility in a generic set of $n$-tuples of utilities on $\Omega$ there are finitely many distributions on $\Omega$ induced by the Nash equilibria of the game given by the induced utilities on $S$. We give a counterexample refuting the conjecture for $n \geq 3$. Several special cases of the conjecture follow from well known theorems, and we provide some generalizations of these results.
\end{abstract}




\title{
On the Generic Finiteness of Equilibrium Outcome Distributions in Game Forms
}

\author{
by \\ Srihari Govindan and Andrew McLennan
}

\section{Introduction}

This paper studies a class of conjectures concerning the generic determinacy of the Nash equilibrium concept. Let $S_{1}, \ldots, S_{n}$ be nonempty finite sets of pure strategies, let $S=S_{1} \times \ldots \times S_{n}$, let $\Omega$ be a finite set of "outcomes," and let $\theta: S \rightarrow \Delta(\Omega)$ be a function, where $\Delta(\Omega)$ is the set of probability measures on $\Omega$. Utility functions $u_{1}, \ldots, u_{n}: \Omega \rightarrow$ $\mathbb{R}$ induce payoff functions $v_{1}, \ldots, v_{n}: S \rightarrow \mathbb{R}$ according to the formula for expected utility: $v_{i}(s)=\sum_{\omega \in \Omega} u_{i}(\omega) \cdot \theta(s)(\omega)$. The most general form of the conjecture (refuted by counterexample in $\S 3$ ) is that for a generic set of $u_{1}, \ldots, u_{n}$ the set of distributions on $\Omega$ induced by Nash equilibria of the derived game is finite. Our counterexample has three agents; whether the conjecture holds for two person games is an open problem.

In the remainder of this introduction we review known results of this type and explain the motivation for the conjecture. Notation and mathematically attractive variants of the conjecture are presented in $\S 2$. In $\S 3$ we present a counterexample which refutes the general form of the conjecture, and we describe some interesting open questions. Useful results from semi-algebraic geometry are presented in $\S 4$, and in $\S 5$ these tools are used to prove the following positive results: (a) the conjecture holds when $\# \Omega=2$; (b) if, at all mixed strategy tuples, for each agent $i$ the set of distributions on $\Omega$ that agent $i$ can induce by changing his strategy is ( $\left.{ }^{\#} S_{i}-1\right)$-dimensional, then for generic utilities there are finitely many equilibria; (c) for $n=2$, generic common interest and zero sum games have finitely many equilibrium outcomes; (d) for a generic set of functions $\theta: S \rightarrow \Delta(\Omega)$ it is the case that, for generic utilities, there are finitely many equilibria.

\subsection{Extensive Games of Perfect Information}

Consider an extensive form game of perfect information (without chance moves), let $Z$ be the set of terminal nodes, and let $\psi: Z \rightarrow \Omega$ be a function from terminal nodes to outcomes. Then

$$
G_{P}=\left\{u \in\left(\mathbb{R}^{\Omega}\right)^{I}: u_{i}\left(\omega_{1}\right) \neq u_{i}\left(\omega_{2}\right) \text { for all } i \in I \text { and all } \omega_{1}, \omega_{2} \in \Omega\right\}
$$

is evidently the complement of a finite union of hyperplanes. For each $i$ let $S_{i}$ be the set of pure strategies for agent $i$, where a pure strategy is a function assigning a successor node to each node at which agent $i$ chooses. Then each $s \in S=S_{1} \times \ldots \times S_{n}$ determines a unique terminal node $\zeta(s) \in Z$ in the usual way, and we may let $\theta=\psi \circ \zeta: S \rightarrow \Omega$. 
We claim that if $u \in G_{P}$ and $\sigma$ is a Nash equilibrium for $u$, then $\mathbf{P}(\sigma)$ is degenerate in the sense of assigning all probability to some element of $\Omega$. To show this we begin by observing that $\sigma$, appropriately interpreted, is a Nash equilibrium of the normal form game obtained, as above, from the extensive form derived by eliminating all nodes and branches that occur with zero probability. In this smaller tree every node occurs with positive probability, so for each node there is a well defined conditional probability distribution on $\Omega$. If there is any node at which this conditional distribution is nondegenerate (i.e. not a vertex of $\Delta(\Omega)$ ), then there must be a "lowest" such node, by which we mean that the conditional distributions at all successor nodes are degenerate. But at such a lowest node the agent who chooses cannot be optimizing, since he or she is not indifferent between any of the pure outcomes resulting from the various successor nodes.

The argument just given is a slight generalization of the one used by Kuhn (1953) to prove his "backwards induction" theorem characterizing what are now called subgame perfect equilibria of games of perfect information.

\subsection{Two Agents, Two Outcomes}

Assume that $n=2$ while $\Omega=\{a, b\}$. Generically, neither agent is indifferent between $a$ and $b$. If the agents disagree about whether $a$ is better than $b$ then, after rescaling, the game is zero sum, and the minimax theorem implies that all Nash equilibria have the same expected payoffs, hence induce the same distribution on $\Omega$. In the case that the agents agree that, say, $a$ is better than $b$, then a slightly more complicated argument is required. Any Nash equilibrium is a totally mixed equilibrium of the game obtained by eliminating unused strategies, and the equilibrium conditions of totally mixed equilibrium are unaffected by reversing an agent's preference of $a$ over $b$, so the result is obtained from the minimax theorem and the fact that the set of possible truncations of the game is finite. In Theorem 5.3 below we show that this result does not depend on the assumption that $n=2$. The assumption $\# \Omega=2$ implies that, generically, either the game is zero sum (up to positive affine rescaling of utilities) or a game of common interest, in that the two agents have the same utility function (again, up to rescaling). Theorem 5.7 shows that this consequence suffices for the result: generically in the space of common interest utilities, and also generically in the space of zero sum payoffs, there are finitely many equilibrium outcomes.

\subsection{Harsanyi's Theorem}

When $\Omega=S$ and $\theta$ is the identity function, our conjecture reduces to the issue of generic finiteness in the space of normal form payoffs. Perhaps motivated by the introduction of issues of generic determinacy in economic theory in the work of Debreu (1970) on "smooth" general equilibrium theory, Harsanyi (1973) proved that, for a generic set of normal form payoffs, it is the case that all equilibria are regular. We do not wish to give 
a precise description of the notion of regular equilibrium. Instead we simply note that a regular equilibrium is necessarily an isolated pointed in the set of equilibria, and since the set of equilibria is always compact, when all equilibria are regular the set of equilibria is necessarily finite. Thus Harsanyi's theorem implies the case of our conjecture given by normal form payoffs. Theorems 5.6 and 5.10 generalize the generic finiteness corollary of Harsanyi's result. It seems plausible that suitable generalizations of Harsanyi's notion of regularity can be defined, and that the framework laid out in $\S 4$ and $\S 5$ would be useful in discussing these notions.

\subsection{Extensive Games of Incomplete Information}

Harsanyi's result can be criticized as being of diminished relevance insofar as the normal form is typically thought of as being derived from an extensive form, so that many normal form pure strategy vectors correspond to a single terminal node of the extensive form. The space of extensive form utilities corresponds to a linear subspace of the space of normal form utilities, and generic finiteness in the subspace does not follow automatically from generic finiteness in the larger space. There are numerous examples of extensive forms with open sets of terminal node utilities, for each of which the set of Nash equilibria is infinite.

Kreps and Wilson (1982) proved a theorem that responds to this critique. Consider an extensive form game of incomplete information, let $Z$ be the set of terminal nodes, and let $\Omega=Z$. Kreps and Wilson (1982) show that for a generic set of $u \in\left(\mathbb{R}^{Z}\right)^{I}$ the set of distributions on $Z$ ("paths") induced by sequential equilibria is finite, but they also mention (p. 881) that this remains true if one considers paths induced by Nash equilibria. (Kuhn's (1953) theorem concerning the equivalence of normal form and behavioral strategies implies that this is correct as a statement about the Nash equilibria of the normal form.) Their result appears to have considerable epistemological significance insofar as the distribution on terminal nodes is precisely what is seen by a "social scientist" who repeatedly observes agents playing the game.

A variant of the criticism above can be leveled at Kreps and Wilson's result. Many games arising in economic models, and in other contexts, have sets of terminal nodes that are naturally regarded as a priori equivalent for reasons arising out of the nature of the phenomenon being modelled. Again, the "relevant" space of terminal node utilities is a linear subspace of the space of all terminal node utilities, and generic finiteness in the subspace does not follow from Kreps and Wilson's theorem. This point of view is argued by Mailath, Samuelson, and Swinkel (1993, p. 283). Were it true, the conjecture presented here would respond to this criticism.

"Cheap talk" formalized by payoff-irrelevant message spaces is a particularly important instance of the type of identification just mentioned. Park (1997) shows that, in sender-receiver games in which an informed sender chooses a message that is observed 
by an uninformed receiver, who then chooses an action, for generic payoffs on the set of (type-of-sender, action) pairs there are finitely many distributions on the set of such pairs induced by Nash equilibria. It is noteworthy that Park does not fix the message space in advance, so that the set of equilibrium distributions on type-action pairs is in effect the union, over message spaces of all cardinalities, of the set of equilibrium pairs for a given message space.

\section{The Conjectures}

The conditions defining Nash equilibrium can be thought of as a system of equalities (for each agent, all strategies assigned positive probability must have the same expected payoff) and weak inequalities (probabilities are nonnegative, and for each agent the expected payoff resulting from any unused strategy is not greater than the expected payoff resulting from the strategies that are used). It seems plausible that the weaker equilibrium notion given by the equalities alone will have the same generic properties, and that the analysis might be simpler. As we explain below, generic finiteness results for this weak notion imply corresponding results for the more demanding concepts. Thus we are led to consider some unusual constructs.

For any finite set $X$ let the affine hull of the standard unit vectors in $\mathbb{R}^{X}$ be

$$
H(X)=\left\{\mu \in \mathbb{R}^{X}: \Sigma_{x \in X} \mu(x)=1\right\} .
$$

Then the set of probability measures on $X$ is $\Delta(X)=H(X) \cap \mathbb{R}_{+}^{X}$, and the set of interior probability measures is $\Delta^{\circ}(X)=H(X) \cap \mathbb{R}_{++}^{X}$. For $x \in X$ we will often let $x$ also denote the Dirac measure at $x$, that is, the probability measure on $X$ that assigns all mass to $x$.

We fix a game form given by the following information. There are $n$ agents indexed by $i=1, \ldots, n$. For each $i$ let $S_{i}$ be a nonempty finite set of pure strategies. Let $S=S_{1} \times \ldots \times S_{n}$ be the set of pure strategy vectors, let $\Omega$ be a finite set of outcomes and let the pure outcome function be $\theta: S \rightarrow \Delta(\Omega)$, a function assigning an outcome to each vector of pure strategies. We say that the game form is a pure outcome game form if the image of $\theta$ is contained in $\Omega$, where we identify $\Omega$ with the set of vertices of $\Delta(\Omega)$.

$$
\text { . Let } \quad \Sigma=\Delta\left(S_{1}\right) \times \ldots \times \Delta\left(S_{n}\right) ; \quad \Sigma^{\circ}=\Delta^{\circ}\left(S_{1}\right) \times \ldots \times \Delta^{\circ}\left(S_{n}\right) ; \quad \Xi=H\left(S_{1}\right) \times \ldots \times H\left(S_{n}\right) .
$$

Typical elements of $\Xi$ are denoted by $\sigma=\left(\sigma_{1}, \ldots, \sigma_{n}\right), \tau=\left(\tau_{1}, \ldots, \tau_{n}\right), \ldots$ For $\sigma \in \Xi$ and $\tau_{i} \in H\left(S_{i}\right)$, let

$$
\sigma \mid \tau_{i}=\left(\sigma_{1}, \ldots, \sigma_{i-1}, \tau_{i}, \sigma_{i+1}, \ldots, \sigma_{n}\right) .
$$

The probabilistic outcome function derived from $\theta$ is $\mathbf{P}: \Xi \rightarrow H(\Omega)$ given by the formula

$$
\begin{gathered}
\mathbf{P}(\sigma)=\sum_{s \in S}\left(\prod_{i=1}^{n} \sigma_{i}\left(s_{i}\right)\right) \cdot \theta(s) . \\
-4-
\end{gathered}
$$


Observe that $\mathbf{P}$ is multiaffine: holding $\sigma_{1}, \ldots, \sigma_{i-1}, \sigma_{i+1} \ldots, \sigma_{n}$ fixed, $\mathbf{P}$ is an affine function of $\sigma_{i}, i=1, \ldots, n$. Conversely, straightforward algebra (see 5.8) shows that if $\mathbf{P}: \Xi \rightarrow H(\Omega)$ is multiaffine, then it coincides with the probabilistic outcome function derived, via the formula above, from the pure outcome function obtained by restricting $\mathbf{P}$ to $n$-tuples of pure strategies.

Let $U_{i}=\left(\mathbb{R}^{\Omega}\right)^{*}$ (the dual of $\left.\mathbb{R}^{\Omega}\right)$ be the space of utilities for agent $i, i=1, \ldots, n$, and let the space of utility vectors be $U=U_{1} \times \ldots \times U_{n}$ with typical element $u=\left(u_{1}, \ldots, u_{n}\right)$. Our analysis involves three notions of (mathematical, not necessarily conceptual) equilibrium:

Definition 2.1: $\sigma \in \Sigma$ is a Nash equilibrium for $u \in U$ if

$$
\sigma_{i}\left(s_{i}\right) \cdot u_{i}\left(\mathbf{P}\left(\sigma \mid s_{i}\right)-\mathbf{P}\left(\sigma \mid t_{i}\right)\right) \geq 0 \text { for all } i=1, \ldots, n \text { and } s_{i}, t_{i} \in S_{i} \text {. }
$$

Let $E_{N}=\{(\sigma, u) \in \Sigma \times U: \sigma$ is a Nash equilibrium for $u\}$. Let $G_{N}$ be the set of $u \in U$ such that $\{\mathbf{P}(\sigma): \sigma$ is a Nash equilibrium for $u\}$ is finite.

Definition 2.2: $\sigma \in \Sigma^{\circ}$ is a totally mixed equilibrium for $u \in U$ if

$$
u_{i}\left(\mathbf{P}\left(\sigma \mid s_{i}\right)-\mathbf{P}\left(\sigma \mid t_{i}\right)\right)=0 \text { for all } i=1, \ldots, n \text { and } s_{i}, t_{i} \in S_{i} .
$$

Let $E_{T}=\left\{(\sigma, u) \in \Sigma^{\circ} \times U: \sigma\right.$ is a totally mixed equilibrium for $\left.u\right\}$. Let $G_{T}$ be the set of $u \in U$ such that $\{\mathbf{P}(\sigma): \sigma$ is a totally mixed equilibrium for $u\}$ is finite.

Definition 2.3: $\sigma \in \Xi$ is a quasiequilibrium for $u \in U$ if

$$
u_{i}\left(\mathbf{P}\left(\sigma \mid s_{i}\right)-\mathbf{P}\left(\sigma \mid t_{i}\right)\right)=0 \text { for all } i=1, \ldots, n \text { and } s_{i}, t_{i} \in S_{i} .
$$

Let $E_{Q}=\{(\sigma, u) \in \Xi \times U: \sigma$ is a quasiequilibrium for $u\}$. Let $G_{Q}$ be the set of $u \in U$ such that $\{\mathbf{P}(\sigma): \sigma$ is a quasiequilibrium for $u\}$ is finite.

Our formulation of the equilibrium condition is somewhat unusual, but it is evident that our definition of Nash equilibrium is standard: expected utility maximization is equivalent to assigning zero probability to any pure strategy whose expected utility is less than that of some other pure strategy. The importance of totally mixed equilibrium arises out of the observation that a Nash equilibrium for a utility $u$ is (in the obvious sense) a totally mixed equilibrium of the game consisting of $u$ and the game form obtained by eliminating all unused pure strategies. The notion of quasiequilibrium is obtained by dropping the requirement that probabilities be positive from the definition of totally mixed equilibrium.

Definition 2.4: For any $m \geq 0$ we say that $G \subset \mathbb{R}^{m}$ is a generic set, or generic, if $\mathbb{R}^{m}$ - (int $G$ ) has Lesbesgue measure 0 . 
The following conjectures are the main concern of this paper.

Conjecture $N: G_{N}$ is a generic set.

Conjecture $T: G_{T}$ is a generic set.

Conjecture $Q: G_{Q}$ is a generic set.

Of course Conjecture $N$ is the proposition of greatest conceptual interest. Obviously it implies Conjecture $T$. On the other hand, for any game form there are finitely many game forms obtained by deleting pure strategies, and finite intersections of generic sets are generic, so if Conjecture $T$ held for all game forms, Conjecture $N$ would follow.

Clearly Conjecture $Q$ would imply Conjecture $T$, and thus Conjecture $N$. One's crude intuition is that the "generic situations" inside and outside the interior of the unit simplex should be the same, so we would not expect Conjecture $Q$ to be false while Conjecture $T$ is true. Conjecture $Q$ is mathematically simpler than Conjecture $T$, so it appears to be the most inviting framework for analysis.

It is conceivable that there is a continuum of equilibrium distributions over $\Omega$, but that all of them give all agents the same expected utility. Consequently, associated with each of these conjectures is the seemingly weaker conjecture that for generic utilities there are finitely many vectors of expected utilities generated by equilibria of the given type. However, unless the number of players is fixed, a counterexample to the strong conjecture can be converted to a counterexample for the weak conjecture by adding a player with only one move who (generically) cares about the variation in outcome allowed by equilibrium. For the case of two agent games the weak conjecture is established by Mas-Colell (1994), the strong form of the conjecture for two agents is an open problem. From this point of view it is important to note that the three agent counterexample in $\S 3$ has a continuum of induced utility vectors.

\section{The Counterexample}

We now refute Conjecture $\mathrm{T}$ (hence also Conjectures $\mathrm{N}$ and $\mathrm{Q}$ ) by means of a counterexample. Consider the following game form in which agent 1 is the row player, agent 2 is the column player, and agent 3 is the matrix player:

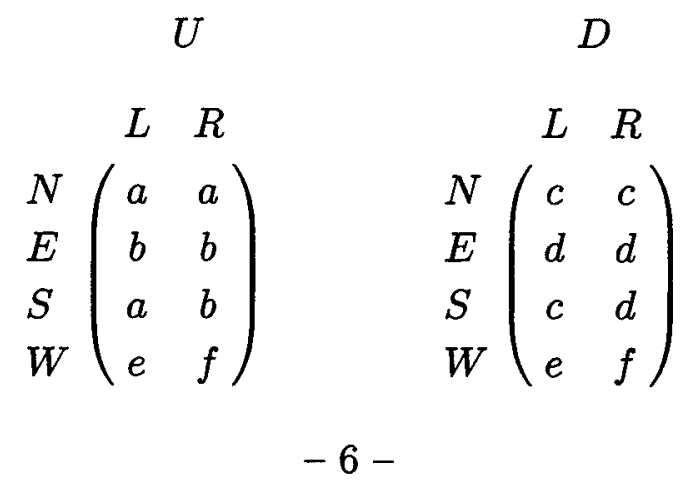


In our discussion we will attach subscripts to the outcomes to indicate payoffs, so that $a_{1}$ is the payoff to agent 1 when outcome $a$ occurs, etc. Let $p, q, r$, and $s$ be the probabilities of $N, E, S$, and $W$, respectively; of course $p+q+r+s=1$. Let $y$ and $z$ be the probabilities of $L$ and $U$ respectively.

We now derive necessary conditions for a totally mixed equilibrium. To begin with note that agent 1's indifference between $N$ and $E$ implies that

$$
z a_{1}+(1-z) c_{1}=z b_{1}+(1-z) d_{1}
$$

so that

$$
z=\frac{d_{1}-c_{1}}{a_{1}-b_{1}-c_{1}+d_{1}} .
$$

It is a very important point that the lottery over outcomes resulting from $S$ is a convex combination of the lotteries resulting from $N$ and $E$, so that whenever agent 1 is indifferent between $N$ and $E$, she is also indifferent between either of these strategies and $S$. On the other hand indifference between $N$ and $W$ amounts to

$$
y e_{1}+(1-y) f_{1}=z a_{1}+(1-z) c_{1}
$$

so that

$$
y=\frac{c_{1}-f_{1}+z\left(a_{1}-c_{1}\right)}{e_{1}-f_{1}} .
$$

For agent 2 indifference between $L$ and $R$ is the condition

$$
r\left(z a_{2}+(1-z) c_{2}\right)+s e_{2}=r\left(z b_{2}+(1-z) d_{2}\right)+s f_{2}
$$

so that

$$
r=s \cdot \frac{\left(f_{2}-e_{2}\right)}{z\left(a_{2}-b_{2}\right)+(1-z)\left(c_{2}-d_{2}\right)} .
$$

For agent 3 indifference between $U$ and $D$ is the condition

$$
p a_{3}+q b_{3}+r\left(y a_{3}+(1-y) b_{3}\right)=p c_{3}+q d_{3}+r\left(y c_{3}+(1-y) d_{3}\right)
$$

so that

$$
p\left(a_{3}-c_{3}\right)+q\left(b_{3}-d_{3}\right)+r\left(y\left(a_{3}-c_{3}\right)+(1-y)\left(b_{3}-d_{3}\right)\right)=0 .
$$

We can now easily produce the desired counterexample. So long as the parameters avoid problems of division by zero in (1) and (2), agent 1's indifference conditions determine $y$ and $z$. Once these numbers are fixed, there are two linear conditions (in addition to $p+q+r+s=1$ ) on agent 1's strategy, with parameters given by the utilities of agents 2 and 3. Except on an obvious set, with positive codimension, of special utilities for these two agents, there will be a one dimensional space of $(p, q, r, s)$ satisfying all conditions. There is 
no great art to choosing particular values of the parameters such that $y, z \in(0,1)$ and this line passes through the interior of the simplex $\left\{(p, q, r, s) \in \mathbb{R}_{+}^{4}: p+q+r+s=1\right\}$, and we leave this aspect of the argument to the reader. The set of solutions varies continuously, in the obvious sense, on the open set of parameters that do not give rise to some division by zero in the equations above, so we have the desired open set of utilities for which there are a continuum of totally mixed equilibria.

When agent 1 plays $W$, the outcomes are different from those resulting from any other pure strategy agent 1 might choose, so the one dimensional continuum of totally mixed equilibria maps to a one dimensional set of distributions over outcomes so long as $s$ varies in the set of equilibria, which is typically the case. Equations (1)-(4) make no reference to $e_{3}$ and $f_{3}$, so the expected utility of agent 3 varies so long as these payoffs are not exceptional.

\section{Semi-algebraic Geometry}

We now state some important concepts and results of semi-algebraic geometry, to some extent indicating their consequences for the problems at hand. Proofs of major results are omitted: the authoritative reference for this material is Bochnak, Coste, and Roy (1987); expositions similar to the one below, but somewhat more extensive, occur in Blume and Zame (1994) and Schanuel, Simon, and Zame (1991).

A real algebraic variety is a set of the form $\left\{x \in \mathbb{R}^{m}: P(x)=0\right\}$, where $P$ is a polynomial in $x_{1}, \ldots, x_{m}$ with real coefficients. Observe that any condition of the form $\left[P_{1}(x)=0\right.$ or $\ldots$ or $\left.P_{k}(x)=0\right]$ is equivalent to $P_{1}(x) \cdot \ldots \cdot P_{k}(x)=0$, while any condition of the form $\left[P_{1}(x)=0\right.$ and $\ldots$ and $\left.P_{k}(x)=0\right]$ is equivalent to $P_{1}(x)^{2}+\ldots+P_{k}(x)^{2}=0$, so the class of real algebraic varieties is closed under finite union and intersection. (In fact the Hilbert basis theorem implies that an arbitrary intersection of real algebraic varieties is a real algebraic variety - cf. Fulton $(1969, \S I .4)$.) Whitney (1957) is a relatively elementary exposition of the basic geometric facts concerning these sets.

A semi-algebraic set is a finite union of sets of the form

$$
\left\{x \in \mathbb{R}^{m}: P(x)=0 \text { and } Q_{1}(x)>0 \text { and } \ldots \text { and } Q_{k}(x)>0\right\},
$$

where $P$ and $Q_{1}, \ldots, Q_{k}$ are polynomials in $x_{1}, \ldots, x_{m}$ with real coefficients. Obviously this class includes sets defined by weak inequalities as well as equations and strict inequalities. This class of sets is also closed under finite unions and finite intersections, and in addition it is closed under complementation.

A $d$-dimensional algebraic partial manifold in $\mathbb{R}^{m}$ is a set $M \subset \mathbb{R}^{m}$ such that for each $p \in M$ there exist polynomials $P_{1}, \ldots, P_{m-d}$ and $U$, a neighborhood (in $\mathbb{R}^{m}$ ) of $p$, such that $D P_{1}(p), \ldots, D P_{m-d}(p)$ are linearly independent (in $\left.\left(\mathbb{R}^{m}\right)^{*}\right)$ and

$$
\begin{gathered}
M \cap U=\left\{q \in U \mid P_{1}(q)=\ldots=P_{m-d}(q)=0\right\} . \\
-8-
\end{gathered}
$$


The codimension of $M$ is $m-d$. If, in addition, $M$ is a semi-algebraic set, then we say that $M$ is a semi-algebraic manifold. Since semi-algebraic sets may have of singularities, which makes analysis on them difficult, the following property of semi-algebraic sets has many consequences.

Proposition 4.1: (Stratification - Whitney (1957), Bochnak, Coste, and Roy (1987, 9.1.8)) If $A$ is a semi-algebraic set, then $A$ is the union of a finite number of disjoint, connected semi-algebraic manifolds $A_{j}$ with $A_{j} \subset c \ell\left(A_{k}\right)$ whenever $A_{j} \cap c \ell\left(A_{k}\right) \neq \emptyset$.

One consequence of this is that we may define $\operatorname{dim} A$, the dimension of $A$, to be the largest dimension of any element of any decomposition as per 4.1. The following special case of 4.1 is a useful way of showing that sets are finite.

Lemma 4.2: If $A$ is a 0 -dimensional semi-algebraic set, then $A$ is finite.

The other extreme dimension is also of interest.

Lemma 4.3: If $G \subset \mathbb{R}^{m}$ is semi-algebraic, then $G$ is generic if and only if $\operatorname{dim}\left(\mathbb{R}^{m}-G\right)<$ $m$.

We will also need the following intuitively obvious fact.

Lemma 4.4: If $A \subset \mathbb{R}^{m}$ and $B \subset \mathbb{R}^{n}$ are semi-algebraic sets, then $\operatorname{dim} A \times B=$ $(\operatorname{dim} A)+(\operatorname{dim} B)$.

Proof: That cartesian products of semi-algebraic manifolds are semi-algebraic manifolds, and that the assertion holds for semi-algebraic manifolds, are more or less immediate consequences of our definitions above. The general case follows from the observation that if $\left\{A_{i}\right\}$ and $\left\{B_{j}\right\}$ are stratifications of $A$ and $B$ in the sense of 4.1, then $\left\{A_{i} \times B_{j}\right\}$ is easily shown to be a stratification of $A \times B$.

If $A$ and $B$ are semi-algebraic sets, then a function $f: A \rightarrow B$ is said to be semialgebraic if its graph is a semi-algebraic set. (Note that this does not imply continuity.) We will make extensive use of the following:

Proposition 4.5: If $f: A \rightarrow B$ is a semi-algebraic function, then $f(A)=\{b \in B: f(a)=b$ for some $a \in A\}$ is a semi-algebraic set.

This is a consequence of one of the most important foundational results of semialgebraic geometry, the Tarski-Seidenberg theorem, which asserts that every first order formula in the theory of real closed fields, e.g. "there exists $y$ such that $x=y^{2}$," is equivalent to a first order formula without quantifiers, e.g. " $x \geq 0$." This result has been exploited by Blume and Zame (1994) and Schanuel, Simon, and Zame (1991) to obtain results concerning, the relationship between perfect and sequential equilibrium, and, respectively, the tracing procedure of Harsanyi and Selten (1988). 
We exploit 4.5 to obtain the following useful fact.

Lemma 4.6: Let $P: \mathbb{R}^{m} \rightarrow \mathbb{R}^{n}$ be a polynomial function, and let $S=\left\{x \in \mathbb{R}^{m}\right.$ : $\left.D P(x)=0 \in \mathrm{L}\left(\mathbb{R}^{m}, \mathbb{R}^{n}\right)\right\}$. Then $P(S)$ is finite.

Proof: By 4.5, $P(S)$ is semi-algebraic, so 4.2 implies that it suffices to show that $P(S)$ is 0-dimensional. If, in some stratification of $P(S)$ as per 4.1 , there is a piece whose dimension is greater that 0 , then there is some linear $\ell: \mathbb{R}^{n} \rightarrow \mathbb{R}$ such that $\ell(P(S))$ has positive dimension. Therefore it suffices to establish the claim when $n=1$, and in this case we can apply Sard's theorem to show that $P(S)$ has measure 0 in $\mathbb{R}^{1}$. That $P(S)$ is 0 -dimensional, hence finite, is now a fairly straightforward consequence of 4.1.

We now present another deep and fundamental theorem of semi-algebraic geometry. Whereas 4.1 decomposes semi-algebraic sets into simple pieces, the following provides a decomposition of maps.

Proposition 4.7: (Generic Local Triviality - Hardt (1980), Bochnak, Coste, and Roy $(1987,9.3 .2)$ ) Let $A$ and $B$ be semi-algebraic sets, and let $g: A \rightarrow B$ be a continuous semi-algebraic function. Then there is a relatively closed semi-algebraic set $B^{\prime} \subset B$ with $\operatorname{dim} B^{\prime}<\operatorname{dim} B$ such that each component $C$ of $B-B^{\prime}$ has the following property: there is a semi-algebraic set $F$ (the "fiber") and a semi-algebraic homeomorphism $h: C \times F \rightarrow$ $g^{-1}(C)$ with $g(h(b, f))=b$ for all $b \in C$ and $f \in F$.

Our arguments in $\S 5$ will apply the following property of semi-algebraic maps. "Space filling curves" show that continuous functions do not have this property.

Lemma 4.8: (Bochnak, Coste, and Roy $(1987,2.8 .8)$ ) If $A$ is a semi-algebraic set and $f: A \rightarrow \mathbb{R}^{m}$ is a semi-algebraic function, then $\operatorname{dim} f(A) \leq \operatorname{dim} A$.

This is almost, but not quite, a consequence of 4.7 , since $f$ is not required to be continuous.

The following two consequences of 4.7 are applied in $\S 5$.

Proposition 4.9: If $A$ and $B$ be semi-algebraic sets and $g: A \rightarrow B$ is a continuous semi-algebraic function, then

$$
\operatorname{dim} B+\min _{b \in B} \operatorname{dim} g^{-1}(b) \leq \operatorname{dim} A \leq \operatorname{dim} B+\max _{b \in B} \operatorname{dim} g^{-1}(b) .
$$

Proof: If $C, F$, and $h$ are as in 4.7, then $\operatorname{dim} g^{-1}(C)=\operatorname{dim} C+\operatorname{dim} F$ by 4.6 and 4.7 , and 4.5 also implies that $\operatorname{dim} g^{-1}(b)=\operatorname{dim} h^{-1}\left(g^{-1}(b)\right)=\operatorname{dim}(\{b\} \times F)=\operatorname{dim} F$ for $b \in C$, so that $\operatorname{dim} g^{-1}(C)=\operatorname{dim} C+\operatorname{dim} g^{-1}(b)$ for $b \in C$. Choosing $C$ such that $\operatorname{dim} C=\operatorname{dim} B$, the first asserted inequality follows since $\operatorname{dim} g^{-1}(C) \leq \operatorname{dim} A$. Note that 4.7 can also be 
applied to $g \mid B^{\prime}$, where $B^{\prime}$ is as in 4.7. Therefore we can also find $C, F$, and $h$ as in 4.7 with $\operatorname{dim} g^{-1}(C)=\operatorname{dim} A$, from which we obtain the second inequality.

Proposition 4.10: If $A$ and $B$ be semi-algebraic sets and $g: A \rightarrow B$ is a continuous semi-algebraic function, then

$$
\operatorname{dim}\left\{b \in B: \operatorname{dim} g^{-1}(b)>\operatorname{dim} A-\operatorname{dim} B\right\}<\operatorname{dim} B .
$$

Proof: The argument is similar to the proof of 4.9 , hence is omitted.

Another consequence of 4.7 is the following fact of obvious interest.

Proposition 4.11: $G_{Q}, G_{N}$, and $G_{T}$ are semi-algebraic sets, hence each is generic if and only if its complement has positive codimension.

Proof: We prove this only for $G_{Q}$; the other proofs are similar. Consider the real algebraic variety

$$
\left\{(\sigma, u, \mathbf{P}(\sigma)):(\sigma, u) \in E_{Q}\right\} \subset \Xi \times U \times H(\Omega) .
$$

Let $A$ be its projection onto $U \times H(\Omega) ; 4.1$ implies that $A$ is a semi-algebraic set. Applying 4.7 to the projection of $A$ onto $U$ shows that

$$
G_{Q}=\{u \in U:\{a \in H(\Omega):(u, a) \in A\} \text { is finite }\}
$$

is a semi-algebraic set.

The final notion we need is that of constructible sets. The class of constructible subsets of an affine space $A$ is the smallest collection of subsets that is closed under complementation and intersection, and contains all sets of the form $\{x: P(x)=0\}$, where $P$ is a polynomial function on $A$. Equivalently, the constructible sets are those given by unquantified logical formulas built up from the elementary formulas ' $P(x)=0$ ', $P$ a polynomial function.

Proposition 4.12: A constructible set is either generic or its complement is generic.

Proof: Let $\mathcal{C}$ be the class of sets that are constructible and are either generic or have generic complement. By Whitney (1957) any set of the form $\{x: P(x)=0\}$ is a finite union of semi-algebraic manifolds. If any of the component manifolds has the same dimension as the underlying affine space, then one can show that $P$ vanishes identically on the space. Thus each $\{x: P(x)=0\}$ is in $\mathcal{C}$. In addition, $\mathcal{C}$ is closed under complementation, obviously, and intersection, by virtue of Whitney's result. The definition of the class of constructible sets does not allow it to contain $\mathcal{C}$ as a proper subclass. 


\section{Positive Results}

Our notation is as in $\S 2$. For $i=1, \ldots, n$ and $\sigma \in \Xi$ let

$$
d_{i}(\sigma)=\operatorname{dim} \mathbf{P}\left(\sigma \mid H\left(S_{i}\right)\right)=\operatorname{rank} \frac{\partial \mathbf{P}}{\partial \sigma_{i}}(\sigma)
$$

be the dimension of the set of elements of $H(\Omega)$ that agent $i$ can achieve by varying his strategy. For each vector $\delta=\left(\delta_{1}, \ldots, \delta_{n}\right)$ of nonnegative integers let $\Xi(\delta)=\{\sigma \in \Xi$ : $\left.d_{i}(\sigma)=\delta_{i}, i=1, \ldots, n\right\}$. We begin by considering strategy vectors that are equilibria for all vectors of utilities.

Definition 5.1: If, for all $i=1, \ldots, n, d_{i}(\sigma)=0$, i.e. $\sigma \in \Xi(0)$, then we say that $\sigma$ is a universal equilibrium point.

Proposition 5.2: $\mathbf{P}(\Xi(0))$ is finite.

Proof: Since $\sigma$ is a universal equilibrium point if and only if $D \mathbf{P}(\sigma)=0$, the claim follows directly from 4.6.

When $\# \Omega=2$, for generic (i.e. everybody cares which outcome occurs) utilities the set of quasiequilibria is precisely the set of universal equilibrium points, so the next result is a corollary of 5.2. It might be thought of as pertaining to contests between two teams in which winning and losing are the only outcomes, but note that we contemplate equilibria for teams without a "coach" in that the notion of equilibrium allows a team's behavior to be collectively suboptimal even though each team member is acting in the best interest of the team, given the strategies of all other agents.

Theorem 5.3: If $\# \Omega=2$, then, for a generic set of utilities $u$, a point $\sigma$ is an equilibrium for $u$ if and only if $\sigma$ is a universal equilibrium point, so that $\{\mathbf{P}(\sigma): \sigma$ is a quasiequilibrium for $u$ \} is finite.

There is a different argument leading to this result. A common interest game is one in which all agents have the same utility function : $u_{1}=\ldots=u_{n}$. Mertens, Sorin, and Zamir (1994, pp. 59-60) point out that an equilibrium for a common utility function $u$-is a $\sigma$ such that $D(u \circ \mathbf{P})(\sigma)=0$, so 4.6 implies that there are only finitely many (common) equilibrium utilities. When ${ }^{\#} \Omega=2$ the conditions of totally mixed equilibrium are unaffected if an agent's utility function is replaced by its negation, so there is no loss of (generic) generality in assuming that the game is one of common interest. Also, when $\# \Omega=2$ each utility level corresponds to a unique outcome.

We now examine the other extreme possibility for $\delta$.

Definition 5.4: We say that $\sigma$ is an maximal dimension point if $d_{i}(\sigma)=\# S_{i}-1$ for all $i=1, \ldots, n$. We say that $\sigma$ is a maximal dimension equilibrium for $u$ if $\sigma$ is an maximal dimension point and a quasiequilibrium for $u$. 
Proposition 5.5: The dimension of $B=\{(\sigma, u): \sigma$ is an maximal dimension equilibrium for $u\}$ is not greater than the dimension of $U$.

Proof: Let $C$ be the set of maximal dimension points in $\Xi$, and let $\pi: B \rightarrow C$ be the natural projection. If $\sigma \in C$, then for each $i$ the set of $u_{i}$ such that the conditions of quasiequilibrium for agent $i$, that is $\left\{u_{i} \in U_{i}: u_{i} \circ D_{i} P(\sigma)=0\right\}$, is $\left(\operatorname{dim} U_{i}-\left({ }^{\#} S_{i}-1\right)\right)$ dimensional. Consequently the dimension of $\pi^{-1}(\sigma)$ is $\sum_{i}\left(\operatorname{dim} U_{i}-\left({ }^{\#} S_{i}-1\right)\right)=\operatorname{dim} U-$ $\operatorname{dim} \Xi$, so the claim follows from 4.9 .

Recall our formulation of Harsanyi's theorem: $\theta: S \rightarrow \Omega$ is a bijection, implying that every $\sigma$ is a maximal dimension point. This turns out to be the crucial element in the proof, as we will see shortly. In a subjective sense at least, for given $S_{1}, \ldots, S_{n}$, there can be "many" identifications of elements of $S=S_{1} \times \ldots \times S_{n}$ with each other before it ceases to be the case that every $\sigma$ is a maximal dimension point. Perhaps the simplest nontrivial example is: $I=\{1,2\}, S_{1}=\left\{s_{1}, t_{1}\right\}, S_{2}=\left\{s_{2}, t_{2}\right\}, \Omega=\{a, b, c\}$, and $\theta$ given by the matrix:

$$
s_{1}\left(\begin{array}{cc}
s_{2} & t_{2} \\
t_{1} & b \\
c & a
\end{array}\right) .
$$

Theorem 5.6: If every $\sigma \in \Xi$ is a maximal dimension point, then

$$
\operatorname{dim}(\{(\sigma, u): \sigma \text { is an quasiequilibrium for } u\}) \leq \operatorname{dim} U,
$$

so that $\{\sigma: \sigma$ is an quasiequilibrium for $u\}$ and $\{\mathbf{P}(\sigma): \sigma$ is an quasiequilibrium for $u\}$ are finite for a generic set of $u$. In particular Conjecture $Q$ holds.

Proof: The first claim follows from 5.5. Applying 4.10 to the projection of $\{(\sigma, u): \sigma$ is an quasiequilibrium for $u\}$ onto $U$ shows that $\{\sigma: \sigma$ is an quasiequilibrium for $u\}$ is 0 -dimensional, hence finite by 4.3 , for all $u$ outside some set of positive codimension. Now apply 4.4 .

If a game form satisfying the hypotheses of 5.6, then any truncation of the game form resulting from the elimination of some pure strategies also satisfies these hypotheses. As explained in $\S 2$, it follows that for such game forms we have the implications

$$
\text { Conjecture } \mathrm{Q} \Rightarrow \text { Conjecture } \mathrm{T} \Rightarrow \text { Conjecture } \mathrm{N} \text {. }
$$

It is possible to generalize Theorem 5.6 along the following lines: for each $n$-tuple of integers $\delta=\left(\delta_{1}, \ldots, \delta_{n}\right)$ with $0 \leq \delta_{i} \leq \# S_{i}-1$, let $\Sigma(\delta)$ be the set of $\sigma$ in which the rank of $\frac{\partial \mathbf{P}}{\partial \sigma_{i}}(\sigma)$ is at least ${ }^{\#} S_{i}-1-\delta_{i}$. If the dimension of $\Sigma(\delta)$ is $\sum_{i}{ }^{\#} S_{i}-1-\delta_{i}$ or less, there are finitely many quasiequilibria. However, this more general hypothesis is not necessarily preserved under truncation, so the finiteness of the set of Nash equilibirum outcomes is not an automatic consequence. 
We now prove that Conjecture Q (and hence also the other conjectures) holds for two subclasses of two person game forms: common interest games, i.e., where $u_{1}=u_{2}$, and zero sum games, where $u_{1}+u_{2}=0$.

Theorem 5.7: Generic two person common interest games, and generic two person zero sum games, have at most a single quasiequilibrium outcome.

Proof: The conditions of quasiequilibrium are unaffected if one agent's utility function is negated, so the two assertions are equivalent. We will phrase the proof in terms of common interest games.

Let $V_{1}, V_{2}$, and $V_{\Omega}$ be the tangent spaces of $H\left(S_{1}\right), H\left(S_{2}\right)$, and $H(\Omega)$ respectively. That is, at each point $\sigma_{1} \in H\left(S_{1}\right)$ we have $T_{\sigma_{1}} H\left(S_{1}\right)=V_{1}$, because we are identifying all such tangent spaces with the linear space derived, in the obvious way, from the affine structure of $H\left(S_{1}\right)$. Observing that the partial derivative $\frac{\partial \mathbf{P}}{\partial \sigma_{1}}\left(\sigma_{1}, \sigma_{2}\right)$ is independent of the first argument, we have functions

$$
\frac{\partial \mathbf{P}}{\partial \sigma_{1}}: H\left(S_{2}\right) \rightarrow L\left(V_{1}, V_{\Omega}\right) \quad \text { and } \quad \frac{\partial \mathbf{P}}{\partial \sigma_{2}}: H\left(S_{1}\right) \rightarrow L\left(V_{2}, V_{\Omega}\right) .
$$

These functions are affine. Similarly, the mixed second partials

$$
\frac{\partial \frac{\partial \mathbf{P}}{\partial \sigma_{1}}}{\partial \sigma_{2}} \in L\left(V_{2}, L\left(V_{1}, V_{\Omega}\right)\right) \quad \text { and } \quad \frac{\partial \frac{\partial \mathbf{P}}{\partial \sigma_{2}}}{\partial \sigma_{1}} \in L\left(V_{1}, L\left(V_{2}, V_{\Omega}\right)\right)
$$

are independent of the point of evaluation, and by Young's theorem they agree under the usual identification of the respective containing spaces.

For a real valued affine functional $u$ on $H(\Omega)$, interpreted as a common payoff for the two agents, let $\mathbf{P}_{u}=u \circ \mathbf{P}: H\left(S_{1}\right) \times H\left(S_{2}\right) \rightarrow \mathbb{R}$. As above, we have functions

$$
\frac{\partial \mathbf{P}_{u}}{\partial \sigma_{1}}: H\left(S_{2}\right) \rightarrow V_{1}^{*} \quad \text { and } \quad \frac{\partial \mathbf{P}_{u}}{\partial \sigma_{2}}: H\left(S_{1}\right) \rightarrow V_{2}^{*}
$$

and linear maps

$$
\frac{\partial \frac{\partial \mathbf{P}_{u}}{\partial \sigma_{1}}}{\partial \sigma_{2}} \in L\left(V_{2}, V_{1}^{*}\right) \quad \text { and } \quad \frac{\partial \frac{\partial \mathbf{P}_{u}}{\partial \sigma_{2}}}{\partial \sigma_{1}} \in L\left(V_{1}, V_{2}^{*}\right)
$$

(Here $V_{1}^{*}$ and $V_{2}^{*}$ are the duals of $V_{1}$ and $V_{2}$.)

A quasiequilibrium for $u$ is a pair $\left(\sigma_{1}^{*}, \sigma_{2}^{*}\right) \in H\left(S_{1}\right) \times H\left(S_{2}\right)$ with

$$
\frac{\partial \mathbf{P}_{u}}{\partial \sigma_{1}}\left(\sigma_{2}^{*}\right)=0 \in V_{1}^{*} \quad \text { and } \quad \frac{\partial \mathbf{P}_{u}}{\partial \sigma_{2}}\left(\sigma_{1}^{*}\right)=0 \in V_{2}^{*}
$$

The set of real valued affine functionals on $H(\Omega)$ for which the set of quasiequilibria is nonempty is semi-algebraic, and applying stratification to this set shows that we may 
assume that its interior, which we denote by $G$, is nonempty, since otherwise the claim holds trivially. For $u \in G$ let

$$
H_{1 u}=\left\{\sigma_{1}^{*} \in H\left(S_{1}\right): \frac{\partial \mathbf{P}_{u}}{\partial \sigma_{2}}\left(\sigma_{1}^{*}\right)=0\right\} \quad \text { and } \quad H_{2 u}=\left\{\sigma_{2}^{*} \in H\left(S_{1}\right): \frac{\partial \mathbf{P}_{u}}{\partial \sigma_{1}}\left(\sigma_{2}^{*}\right)=0\right\}
$$

Then $H_{1 u} \times H_{2 u}$ is the set of quasiequilibria for $u$. Let $K_{1 u} \subset V_{1}$ be the kernel of $\frac{\partial \frac{\partial \mathbf{P}_{u}}{\partial \sigma_{2}}}{\partial \sigma_{1}}$, and let $K_{2 u} \subset V_{2}$ be the kernel of $\frac{\partial \frac{\partial \mathbf{P}_{u}}{\partial \sigma_{1}}}{\partial \sigma_{2}}$. Then, for any quasiequilibrium $\left(\sigma_{1}^{*}, \sigma_{2}^{*}\right)$, $H_{1 u}=\sigma_{1}^{*}+K_{1 u}$ and $H_{2 u}=\sigma_{2}^{*}+K_{2 u}$.

The map $\mathbf{P}$ may be described by a three dimensional array of numbers whose $\left(s_{1}, s_{2}, \omega\right)$-entry is the probability of $\omega$ when agent 1 plays $s_{1}$ and agent 2 plays $s_{2}$. Each $u \in G$ induces a matrix $A(u)$ with rows indexed by $S_{1}$, columns indexed by $S_{2}$, and $\left(s_{1}, s_{2}\right)$ entry $u\left(\mathbf{P}\left(s_{1}, s_{2}\right)\right)$ that is, numerically, the inner product of the vector $\mathbf{P}\left(s_{1}, s_{2}, \cdot\right)$ with $u$.

Regarding the determinant of each square submatrix of $A(u)$ as an algebraic function of $u$, we see that for each $k^{\prime}=0,1,2, \ldots$ the set of $u$ for which $A(u)$ has $\operatorname{rank} k^{\prime}$ is constructible. We let $k$ be the integer for which this set is generic (cf. Proposition 4.12). Since rank is a lower semicontinuous function on the space of matrices, $k$ is the maximal rank of $A(u)$. Let $G^{*}$ be the set of $u \in G$ for which $A(u)$ has rank $k$.

When $A(u)$ has rank $k, K_{1 u}$ is the intersection of $V_{1}$ with the kernel of $A(u)$, interpreted as an element of $L\left(\mathbb{R}^{S_{1}},\left(\mathbb{R}^{S_{2}}\right)^{*}\right)$. The equilibrium conditions are unaffected if a constant is added to each component of $u$. Therefore we may disregard the possibility that, for generic $u$, there are a continuum of equililibrium outcomes associated with equilibria in which the expected payoff is 0 , since, were this the case, it would follow that, for generic $u$, there were also a continuum of equilibrium outcomes associated with equilibria in which the expected payoff is nonzero. In order for there to be a quasiequilibrium with nonzero expected utility for agent 1 , it must be possible for agent 2 to make agent 1 indifferent among all pure strategies, which amounts to the condition that $(1, \ldots, 1) \in \mathbb{R}^{S_{1}}$ is a linear combination of the columns of $A(u)$. In particular, the kernel of $A(u)$ is contained in $V_{1}$, so for $u \in G^{*}$ we have $\operatorname{dim} K_{1 u}=\operatorname{dim} H_{1 u}=\left|S_{1}\right|-k$.

Fix $u \in G^{*}$ and $\sigma_{2}^{*} \in H_{1 u}$. Consider the correspondence that assigns to each $u \in G^{*}$ the set $H_{2 u}$. Applying stratification to decompose the graph of this correspondence as a finite union of manifolds, then applying Sard's theorem to the projection of each manifold onto $H(\Omega)$, we find that we may assume that any neighborhood of $\left(u, \sigma_{2}^{*}\right)$, in the graph of the correspondence, projects onto a neighborhood of $u$.

Let $\tilde{H}_{1}$ be an affine subspace of $H\left(S_{1}\right)$ that is complementary to $K_{1 u}$ in the sense that

$$
\operatorname{dim} \tilde{H}_{1}+\operatorname{dim} K_{1 u}=\operatorname{dim} H\left(S_{1}\right) \quad \text { and } \quad \tilde{H}_{1}+K_{1 u}=H\left(S_{1}\right)
$$

Then $\operatorname{dim} \tilde{H}_{1}=k-1$. Let $\tilde{V}_{1}$ be the tangent space of $\tilde{H}_{1}$. Consider the map $\tilde{q}: G^{*} \times$ $H\left(S_{2}\right) \rightarrow \tilde{V}_{1}^{*}$ given by $\tilde{q}\left(u, \sigma_{2}^{*}\right)=\frac{\partial \mathrm{P}_{u}}{\partial \sigma_{1}}\left(\sigma_{2}^{*}\right) \mid \tilde{V}_{1}$. The construction of $\tilde{H}_{1}$ implies that $\left(u, \sigma_{2}^{*}\right)$ 
is a regular point of $\tilde{q}$, so the implicit function theorem implies that $\left(u, \sigma_{2}^{*}\right)$ has a neighborhood in which $\tilde{q}^{-1}(0)$ is a manifold whose dimension is $|\Omega|+\left(\left|S_{2}\right|-1\right)-(k-1)=|\Omega|+\left|S_{2}\right|-k$.

Now consider the map $q: G^{*} \times H\left(S_{2}\right) \rightarrow V_{1}^{*}$ given by $q\left(u, \sigma_{2}^{*}\right)=\frac{\partial \mathbf{P}_{u}}{\partial \sigma_{1}}\left(\sigma_{2}^{*}\right)$. Near $\left(u, \sigma_{2}^{*}\right)$ the dimension of $q^{-1}(0)$ must be at least $|\Omega|+\left|S_{2}\right|-k$, since, for $u^{\prime}$ near $u$, the "fiber" $q^{-1}(0) \cap\left(\left\{u^{\prime}\right\} \times H\left(S_{2}\right)\right)$ has dimension $\left|S_{2}\right|-k$. If the rank of $D q\left(u, \sigma_{2}^{*}\right)$ exceeded $k$, we could use the implicit function theorem to display $q^{-1}(0)$, near $\left(u, \sigma_{2}^{*}\right)$, as a subset of a manifold whose dimension is less than $|\Omega|+\left|S_{2}\right|-k$. But the rank of $D q\left(u, \sigma_{2}^{*}\right)$ is at least at large as the rank of $D \tilde{q}\left(u, \sigma_{2}^{*}\right)$, so it is exactly $k$.

Let $B\left(\sigma_{2}^{*}\right)$ be the $\left|S_{1}\right| \times|\Omega|$ matrix whose $\left(s_{1}, \omega\right)$-entry is the weight on $\omega$ in $\mathbf{P}\left(s_{1}, \sigma_{2}^{*}\right)$. Then $\left[B\left(\sigma_{2}^{*}\right) A(u)\right]$ is the matrix of $D q\left(u, \sigma_{2}^{*}\right)$, and its rank is $k$, so $\left|S_{1}\right|-k$ of its rows are linear combinations of the other $k$ rows. (In fact they are affine combinations, since the sum of the entries in each row of $B\left(\sigma_{2}^{*}\right)$ is one.) It follows that the kernel of $B\left(\sigma_{2}^{*}\right)^{T}$ (thought of as acting on column vectors in $\mathbb{R}^{S_{1}}$ ) coincides with the kernel of $A(u)^{T}$. But $\frac{\partial \mathbf{P}}{\partial \sigma_{1}}\left(\sigma_{2}^{*}\right) \in L\left(V_{1}, V_{\Omega}\right)$ and $\frac{\partial \frac{\partial \mathbf{P}_{u}}{\partial \sigma_{2}}}{\partial \sigma_{1}} \in L\left(V_{1}, V_{2}\right)$ are the restrictions of $B\left(\sigma_{2}^{*}\right)^{T}$ and $A(u)^{T}$ to $V_{1}$, so their kernels must also coincide.

This means that $\mathbf{P}\left(\sigma_{1}, \sigma_{2}^{*}\right)=\mathbf{P}\left(\sigma_{1}^{*}, \sigma_{2}^{*}\right)$ for all $\sigma_{1} \in H_{1 u}=\sigma_{1}^{*}+K_{1 u}$, since $\sigma_{1}-\sigma_{1}^{*}$ is in the kernel of $\frac{\partial \mathbf{P}}{\partial \sigma_{1}}\left(\sigma_{2}^{*}\right) \in L\left(V_{1}, V_{\Omega}\right)$. But $\left(\sigma_{1}^{*}, \sigma_{2}^{*}\right)$ is an arbitrary point in the set of quasiequilibria, and the argument holds with agents reversed, so we are done.

Our last result is that, for generic choice of $\theta: S \rightarrow \Delta(\Omega)$ it is the case, for generic $u$, that there are finitely many quasiequilibria for $u$ given $\theta$. This can also be viewed as a generalization of Harsanyi's theorem (when ${ }^{\#} \Omega \geq{ }^{\#} S$ the vectors $\theta(s)$ are linearly independent for generic $\theta$ ), but it is definitely vulnerable to the critique discussed in $\S 1.4$ insofar as this notion of genericity ignores any a priori relations between the various elements of $S$. It seems more interesting from a mathematical point of view, asserting that for any $S_{1}, \ldots, S_{n}$ and $\Omega$ the set of $\theta: S \rightarrow \Delta(\Omega)$ that violate Conjecture Q is "thin."

We now develop the elementary algebra of multiaffine functions, mainly in order to introduce some useful notation.

Lemma 5.8: Suppose we are given a basis $\left\{\tau_{i}^{1}, \ldots, \tau_{i}^{\#} S_{i}\right\} \subset H\left(S_{i}\right)$ of $\mathbb{R}^{S_{i}}$ for each $i=1, \ldots, n$. Suppose also that for each $n$-tuple $\left(j_{1}, \ldots, j_{n}\right)$ with $1 \leq j_{i} \leq \# S_{i}, i=$ $1, \ldots, n$, we are given a point $\mathbf{q}\left(j_{1}, \ldots, j_{n}\right) \in H(\Omega)$. Then the unique multiaffine (with respect to the factorization $\left.\Xi=H\left(S_{1}\right) \times \ldots \times H\left(S_{n}\right)\right)$ function $\mathbf{Q}: \Xi \rightarrow H(\Omega)$ with $\mathbf{Q}\left(\tau_{1}^{j_{1}}, \ldots, \tau_{n}^{j_{n}}\right)=\mathbf{q}\left(j_{1}, \ldots, j_{n}\right)$ for all $n$-tuples $\left(j_{1}, \ldots, j_{n}\right)$ is:

$$
\mathbf{Q}(\sigma)=\sum_{j_{1}=1}^{\# S_{1}} \ldots \sum_{j_{n}=1}^{\# S_{n}}\left(\prod_{i=1}^{n} a_{i}^{j_{i}}\right) \cdot \mathbf{q}\left(j_{1}, \ldots, j_{n}\right) .
$$

Proof: Applying the distributive law appropriately, it is easy to show that the formula above defines a multiaffine function. To establish the converse consider $\sigma \in \Xi$. For 
each $i$ there is a unique ${ }^{\#} S_{i}$-tuple $\left(a_{i}^{1}, \ldots, a_{i}^{\#} S_{i}\right)$ such that $\sigma_{i}=\sum_{j} a_{i}^{j} \tau_{i}^{j}$, and we must have $\sum_{j} a_{i}^{j}=1$ since $\tau_{i}^{1}, \ldots, \tau_{i}^{\#} S_{i}, \sigma_{i} \in H\left(S_{i}\right)$. If $\mathbf{Q}$ satisfies the conditions in the hypotheses, it is affine as function of $\sigma_{1}$, so

$$
\mathbf{Q}(\sigma)=\sum_{j_{1}=1}^{\#} S_{i} a_{1}^{j_{1}} \mathbf{Q}\left(\tau_{1}^{j_{1}}, \sigma_{2}, \ldots, \sigma_{n}\right) .
$$

Iterating this computation leads to the asserted formula.

Insofar as we are now allowing $\theta$ to vary, it is convenient to let $\mathbf{P}_{\theta}$ denote the probabilistic outcome function derived from the pure outcome function $\theta$. Given a pure outcome function $\theta, \sigma \in \Xi$, and $i=1, \ldots, n$, let $d_{i}(\theta, \sigma)=\operatorname{dim} \mathbf{P}_{\theta}\left(\sigma \mid H\left(S_{i}\right)\right)=\operatorname{rank} \frac{\partial \mathbf{P}_{\theta}}{\partial \sigma_{i}}(\sigma)$, and let $d(\theta, \sigma)=\left(d_{1}(\theta, \sigma), \ldots, d_{n}(\theta, \sigma)\right)$. For each $n$-tuple of integers $\delta=\left(\delta_{1}, \ldots, \delta_{n}\right)$ with $0 \leq \delta_{i} \leq \min \left\{\# S_{i}-1,{ }^{\#} \Omega-1\right\}, i=1, \ldots, n$, let

$$
\begin{gathered}
A(\delta)=\left\{(\theta, \sigma) \in H(\Omega)^{S} \times \Xi: d(\theta, \sigma)=\delta\right\}, \text { and let } \\
B(\delta)=\left\{(\theta, \sigma, u) \in H(\Omega)^{S} \times \Xi \times U:(\theta, \sigma) \in A(\delta)\right. \text { and } \\
\sigma \text { is a quasiequilibrium for } u \text { given } \theta\} .
\end{gathered}
$$

Lemma 5.9: For $\delta=\left(\delta_{1}, \ldots, \delta_{n}\right)$ as above we have

$$
\begin{aligned}
& \operatorname{dim} A(\delta)=\operatorname{dim} H(\Omega)^{S}+\operatorname{dim} \Xi-\sum_{i}\left({ }^{\#} \Omega-1-\delta_{i}\right)\left({ }^{\#} S_{i}-1-\delta_{i}\right) \quad \text { and } \\
& \operatorname{dim} B(\delta)=\operatorname{dim} H(\Omega)^{S}+\operatorname{dim} U-\sum_{i}\left({ }^{\#} \Omega-1-\left(\delta_{i}+1\right)\right)\left({ }^{\#} S_{i}-1-\delta_{i}\right) .
\end{aligned}
$$

Proof: Fix $\sigma \in \Xi$. For each $i=1, \ldots, n$ choose a basis $\left\{\tau_{i}^{1}, \ldots, \tau_{i}^{\#} S_{i}\right\} \subset H\left(S_{i}\right)$ of $\mathbb{R}^{S_{i}}$ with $\tau_{i}^{1}=\sigma_{i}$. For each $i$ and $\theta, \mathbf{P}_{\theta}\left(\sigma \mid H\left(S_{i}\right)\right)$ is the affine hull of $\left\{\mathbf{P}_{\theta}\left(\sigma \mid \tau_{i}^{j_{i}}\right): j_{i}=\right.$ $\left.1, \ldots,{ }^{\#} S_{i}\right\}$. We will now use 5.8 to argue that, for the given $\sigma$,

$$
\cdot \operatorname{dim}\left\{\theta \in H(\Omega)^{S}: d(\theta, \sigma)=\delta\right\}=\operatorname{dim} H(\Omega)^{S}-\sum_{i}\left({ }^{\#} \Omega-1-\delta_{i}\right)\left({ }^{\#} S_{i}-1-\delta_{i}\right) .
$$

We will think about constructing an element $\theta$ in terms of choosing the various vectors $\mathbf{P}_{\theta}\left(\tau_{1}^{j_{1}}, \ldots, \tau_{n}^{j_{n}}\right)$, as per 5.8. We may begin by choosing $\mathbf{P}_{\theta}(\sigma)$ arbitrarily, giving us $\#_{\Omega}-1$ dimensions. Given $\mathbf{P}_{\theta}(\sigma)$, for each $i$ we may choose $\delta_{i}$ of the vectors $\mathbf{P}_{\theta}\left(\sigma \mid \tau_{i}^{j_{2}}\right), \ldots, \mathbf{P}_{\theta}\left(\sigma \mid \tau_{i}^{j_{\#} S_{i}}\right)$ arbitrarily, giving us $\delta_{i}(\# \Omega-1)$ dimensions, with the remaining ${ }^{\#} S_{i}-1-\delta_{i}$ vectors constrained to lie in the affine hull of

$$
\left\{\mathbf{P}_{\theta}(\sigma), \mathbf{P}_{\theta}\left(\sigma \mid \tau_{i}^{j_{2}}\right), \ldots, \mathbf{P}_{\theta}\left(\sigma \mid \tau_{i}^{j_{\# S_{i}}}\right)\right\}
$$


giving us another $\left({ }^{\#} S_{i}-\delta_{i}-1\right) \delta_{i}$ dimensions. The remaining ${ }^{\#} S-\sum_{i}\left({ }^{\#} S_{i}-1\right)-1$ vectors $\mathbf{P}_{\theta}\left(\tau_{1}^{j_{1}}, \ldots, \tau_{n}^{j_{n}}\right)\left(j_{i} \neq 1\right.$ for at least two $\left.i\right)$ can also be chosen arbitrarily, giving us ( $\left.{ }^{\#} S-\sum_{i}\left({ }^{\#} S_{i}-1\right)-1\right)\left({ }^{\#} \Omega-1\right)$ dimensions. Adding up all these dimensions (or observing that for each $i$ there are ${ }^{\#} S_{i}-1-\delta_{i}$ constrained vectors, each constrained to lie in an affine subspace of codimension ${ }^{\#} \Omega-1-\delta_{i}$ ), we obtain $(*)$. (This argument is rather informal, but with a little thought the reader should be able to see that 4.9 could be used to develop a fully rigorous version.) The first assertion is now derived from 4.9 applied to the projection of $A(\delta)$ onto $\Xi$. For each $(\theta, \sigma) \in A(\delta)$ we obviously have

$$
\operatorname{dim}\{u \in U: \sigma \text { is a quasiequilibrium for } u \text { given } \theta\}=\Sigma_{i}\left({ }^{\#} \Omega-\delta_{i}\right),
$$

and the second assertion is now obtained from the first by applying 4.9 to the projection of $B(\delta)$ onto $A(\delta)$, observing that $\operatorname{dim} U=n \cdot{ }^{\#} \Omega$ while $\operatorname{dim} \Xi=\sum_{i}\left({ }^{\#} S_{i}-1\right)$, and rearranging algebraicly.

Theorem 5.10: For a generic set of $\theta \subset H(\Omega)^{S}$ it is the case for a generic set of $u \in U$ that $\{\sigma \in \Xi: \sigma$ is a quasiequilibrium for $u$ given $\theta\}$ is finite.

Proof: Insofar as there are finitely many vectors of integers $\delta$ with $0 \leq \delta_{i} \leq \min \left\{\# S_{i}-\right.$ $\left.1,{ }^{\#} \Omega-1\right\}, i=1, \ldots, n$, it suffices to show that, given any such $\delta$, for a generic set of $\theta \in H(\Omega)^{S}$ it is the case for a generic set of $u \in U$ that $\{\sigma \in \Xi:(\theta, \sigma, u) \in B(\delta)\}$ is finite. If $\delta_{i}=\# \Omega-1$ for some $i$ and $(\theta, \sigma, u) \in B(\delta)$, then agent $i$ must be indifferent between all elements of $\Omega$. The set of such utilities $u_{i} \in U_{i}$ is one dimensional, so its complement is generic (recall that $\# \Omega \geq 2$ ), hence we may assume that $\delta_{i}<\# \Omega-1$ for all $i$. When we apply 4.10 to the projection of $B(\delta)$ onto $H(\Omega)^{S}$, using the dimension given in 5.9, we find that

$$
\operatorname{dim}\{(\sigma, u):(\theta, \sigma, u) \in B(\delta)\} \leq \operatorname{dim} U-\sum_{i}\left({ }^{\#} \Omega-1-\left(\delta_{i}+1\right)\right)\left({ }^{\#} S_{i}-1-\delta_{i}\right),
$$

for a generic set of $\theta \in H(\Omega)^{S}$. When we fix $\theta$ such that this is the case and apply 4.10 to the projection of the set above onto $U$, we find that, for generic $u$,

$$
\operatorname{dim}\{\sigma:(\theta, \sigma, u) \in B(\delta)\} \leq-\sum_{i}\left({ }^{\#} \Omega-1-\left(\delta_{i+1}\right)\right)\left({ }^{\#} S_{i}-1-\delta_{i}\right) \leq 0 .
$$

The final inequality in the proof above shows that the argument yields quite a bit more information than is given in the statement of 5.10. Rather than lay this information out systematically (which would be easy enough, but tedious) we draw the reader's attention to one interesting consequence.

Corollary 5.11: If $0 \leq \delta_{i} \leq \min \left\{{ }^{\#} S_{i}-1, \# \Omega-1\right\}, i=1, \ldots, n$, with $\delta_{i} \notin\left\{{ }^{\#} S_{i}-\right.$ $1, \# \Omega-2\}$ for some $i$, then for a generic set of $\theta \in H(\Omega)^{S}$ it is the case for a generic set of $u \in U$ that $\{\sigma \in \Xi(\delta): \sigma$ is a quasiequilibrium for $u$ given $\theta\}$ is empty. 
Proof: In the proof of 5.10 we saw that this assertion holds whenever $\delta_{i}={ }^{\#} \Omega-1$ for some $i$, and if $\delta_{i}<\min \left\{{ }^{\#} S_{i}-1, \# \Omega-2\right\}$ for some $i$, then the argument above shows that for a generic set of $\theta \in H(\Omega)^{S}$ it is the case that, for generic $u$,

$$
\operatorname{dim}\{\sigma:(\theta, \sigma, u) \in B(\delta)\} \leq-\sum_{i}\left({ }^{\#} \Omega-1-\left(\delta_{i}+1\right)\right)\left({ }^{\#} S_{i}-1-\delta_{i}\right)<0
$$




\section{References}

Blume, L.E. and W.R. Zame, (1994), "The Algebraic Geometry of Perfect and Sequential Equilibrium," Econometrica, 62, 795-817.

Bochnak, J., M. Coste, and M-F. Roy, (1987), Géométrie Algébrique Réelle, Berlin: Springer-Verlag.

Debreu, G., (1970), "Economies with a Finite Set of Equilibria," Econometrica, 38, 387392.

Fulton, W., (1969), Algebraic Curves, an Introduction to Algebraic Geometry, New York: Benjamin.

Hardt, R., (1980), "Semi-algebraic Local Triviality in Semi-algebraic Mappings," American Journal of Mathematics, 102, 291-302.

Harsanyi, J.C., (1973), "Oddness of the Number of Equilibrium Points: a New Proof," International Journal of Game Theory, 2, 235-250.

Harsanyi, J.C. and R. Selten, (1988), A General Theory of Equilibrium Selection in Games, Cambridge: MIT Press.

Kreps, D. and R. Wilson, (1982), "Sequential Equilibrium," Econometrica, 50, 863-894.

Kuhn, H.W., (1953), "Extensive Games and the Problem of Information," Annals of Mathematical Studies, 28, 193-216.

Mailath, G.J., L. Samuelson, and J. Swinkels, (1993), "Extensive Form Reasoning in Normal Form Games," Econometrica, 61, 273-302.

Mas-Colell, A., (1994), "Generic finiteness of equilibrium payoffs for bimatrix games," mimeo, Harvard University.

Mertens, J.-F., S. Sorin, and S. Zamir, (1994) "Repeated Games: Part A Background Material", CORE Discussion Paper 9420.

Park, I.-U., (1997), "Generic Finiteness of Equilibrium Outcome Distributions for SenderReceiver Cheap-Talk Games," forthcoming in Journal of Economic Theory.

Schanuel, S.H., L.K. Simon and W.R. Zame, (1991), "The Algebraic Geometry of Games and the Tracing Procedure," in Game Equilibrium Models, Vol. II: Methods, Morals, and Markets, ed. by R. Selten. Berlin: Springer-Verlag.

Whitney, H., (1957), "Elementary Structure of Real Algebraic Varieties," Annals of Mathematics, 66, 545-556. 